\title{
Antimaláricos e Ototoxicidade $e^{(*)}$
}

\section{Antimalarials and Ototoxicity}

\author{
Marcelo Cardoso Figueiredo ${ }^{(1)}$, Ciríaco Cristóvão C. T. Atherino ${ }^{(2)}$, \\ Clarice Viana Monteiro ${ }^{(3)}$ e Roger Abramino Levy ${ }^{(4)}$
}

\section{RESUMO}

Os antimaláricos, como o difosfato de cloroquina, têm sido usados amplamente no tratamento não só da malária, mas também de doenças reumatológicas como a síndrome de Sjögren (SS), artrite reumatóide (AR) e lúpus eritematoso sistêmico (LES). Essas drogas são usadas cronicamente e, em conseqüência do acúmulo nos melanócitos, podem causar hiperpigmentação cutânea, retinopatia e lesão no ouvido interno. Como o protocolo do uso de antimaláricos só envolve a avaliação oftalmológica e das enzimas hepáticas, esta revisão discute a necessidade de novos estudos da avaliação periódica da audição desses pacientes.

Palavras-chave: antimaláricos, cloroquina, ototoxicidade.

\section{INTRODUÇÃO}

A ototoxicidade medicamentosa pode ser definida como uma perturbação transitória ou definitiva da função auditiva e/ou vestibular, induzida por substâncias de uso terapêutico $^{(1)}$. O antimalárico original quinino é derivado da árvore Cinchona, um membro da família Rubiaceae. Seu uso foi descrito pelos incas no tratamento das febres tropicais ${ }^{(2)}$ Os antimaláricos foram sintetizados inicialmente nos EUA durante a II Guerra Mundial, para uso contra a malária pela escassez de quinina, já que o Japão dominou Java, a principal fonte da substância natural na época ${ }^{(2)}$. Sabe-se que, embora a atividade antimalárica da hidroxicloroquina (HCQ) seja a mesma do fosfato de cloroquina (FC), seu potencial tóxico é significativamente menor.

\begin{abstract}
Antimalarials such as chloroquine diphosphate have been widely used not only for the treatment of malaria, but also for several rheumatic diseases such as Sjögren's syndrome (SS), rheumatoid arthritis (RA) and Systemic Lupus Erythematosus (SLE). These drugs are used on a long-term basis and, due to melanocytes' accumulation, can cause cutaneous hyperpigmentation, retinopathy and internal ear damage. As the antimalarials' user follow-up protocol recommends only periodic eye exams and liver function testing, we reviewed the literature questioning whether new studies on the periodic hearing evaluation are required for antimalarials' users.
\end{abstract}

Keywords: antimalarials, chloroquine, ototoxicity.

O FC, um derivado sintético de quinolona, é distribuído gratuitamente em vários Estados do Brasil e amplamente utilizado no tratamento de doenças reumáticas, como a artrite reumatóide (AR), síndrome de Sjögren (SS), e lúpus eritematoso sistêmico (LES) e discóide. O seu potencial ototóxico é conhecido, porém pouco estudado ${ }^{(3)}$. Já a HCQ raramente causa ototoxicidade ${ }^{(4)}$. Efeitos colaterais com o uso de antimaláricos, principalmente FC, como hiperpigmentação cutânea e retinopatia não são incomuns ${ }^{(5)}$. $\mathrm{O}$ protocolo do uso dos antimaláricos envolve a avaliação oftalmológica periódica para detecção precoce de seu acúsmulo na retina, que acarreta uma maculopatia. Os níveis de enzimas hepáticas também são avaliados periodicamente com o intuito de detectar precocemente os sinais de toxicidade hepática. Tanto o acúmulo na retina de FC como a

\footnotetext{
* Disciplinas de Otorrinolaringologia e Reumatologia do Hospital Universitário Pedro Ernesto, Universidade do Estado do Rio de Janeiro (UERJ). Recebido em 12/02/2004. Aprovado, após revisão, em 04/03/2004.

1. Médico residente de Otorrinolaringologia do Hospital Universitário Pedro Ernesto, UERJ.

2. Professor adjunto da disciplina de Otorrinolaringologia do Hospital Universitário Pedro Ernesto, UERJ.

3. Médica residente de Otorrinolaringologia do Hospital Universitário Pedro Ernesto, UERJ.

4. Professor adjunto da disciplina de Reumatologia do Hospital Universitário Pedro Ernesto, UERJ. 
heptotoxicidade, se detectados na fase inicial, podem ser reversíveis com a suspensão do medicamento. O comprometimento auditivo, no entanto, tem sido ignorado e raramente investigado. $\mathrm{O}$ conhecimento mais profundo dos mecanismos de ação e dos efeitos no aparelho auditivo é necessário, para que os pacientes usuários de tais medicações se beneficiem dos meios de prevenção mais eficazes.

\section{AÇÃO DO FOSFATO DE CLOROQUINA (FC) NO OUVIDO INTERNO}

O medicamento é quase completamente absorvido pelo trato gastrintestinal, alcançando rapidamente altas concentrações plasmáticas. Pouca quantidade é excretada, sendo a maior parte depositada nos tecidos. Por esta razão, o desaparecimento de sua ação após a retirada é lento. Um estudo realizado em ratos, demonstrou alta concentração de FC nos tecidos que contêm melanina no ouvido interno, correspondentes à estria vascular, modíolo, plano semilunatum, paredes do saco e do utrículo e canais semicirculares ${ }^{(6)}$. Grande deposição de FC foi encontrada nessas estruturas até 13 dias após a injeção, e mesmo após um ano da administração. A melanina é encontrada no ouvido interno em áreas ricamente vascularizadas, e os melanócitos geralmente circundam os vasos sangüíneos. Desta forma, acredita-se que o acúmulo de FC causaria injúria vascular e mudanças degenerativas na estria vascular e no plano semilunatum. As lesões vasculares e dos tecidos epiteliais podem resultar em uma mudança na composição da endolinfa, o que acarretaria a lesão dos receptores celulares. A lesão primária ototóxica parece ser nos tecidos secretórios e reabsortivos e não nas células receptoras. $\mathrm{O}$ acúmulo e a retenção em longo prazo de FC nas células contendo melanócitos no ouvido interno podem explicar a instalação tardia das lesões e a relação com altas doses cumulativas em lesões ototóxicas $^{(6)}$. Murkherjee relatou um caso de disfunção cócleovestibular grave após uma série de injeções de FC, na qual foi instituída prontamente a terapia com esteróides e vasodilatadores com melhora da audição alcançando um nível socialmente aceitável. Nesse caso, foi sugerido como causa um espasmo de parte das artérias cocleares, provocando diminuição do fluxo sangüíneo para a cóclea. Os medicamentos utilizados por seus efeitos antiinflamatórios, e por controlar a reação de hipersensibilidade dos vasos ao FC, restauraram o suprimento sangüíneo para o ouvido interno ${ }^{(7)}$.

Hart e Naunton ${ }^{(8)}$ relataram, em 1964, dois casos de paresia cócleo-vestibular importante em recém-nato de uma mãe tratada com FC durante a gravidez. Matz e Naunton ${ }^{(9)}$ estudaram, em 1968, o ouvido interno de um natimorto de mãe que fez uso da FC durante a gravidez, e encontraram ausência de células ciliares e redução no número de células de sustentação e células ganglionares, porém, não encontraram alterações na estria vascular tampouco em estruturas labirínticas. Pode-se questionar se haveriam efeitos ototóxicos diferentes quando o FC sofre a primeira passagem pela placenta. Já a HCQ não atravessa a barreira placentária e é utilizada livremente em gestantes com LES na maioria dos centros especializados ${ }^{(10-13)}$. De fato, um estudo duplo-cego, controlado em gestantes com LES, mostrou vantagens nas mães que usaram HCQ sem efeitos adversos ou teratogêncios ${ }^{(14)}$.

\section{MANIFESTAÇÕES CLÍNICAS}

Os sintomas da ototoxicidade relacionados com o uso do FC são semelhantes aos provocados pelos aminoglicosídeos, sendo os principais: zumbidos, perda auditiva neurossensorial e vertigens. A perda auditiva é mais comum de forma leve a moderada e simétrica. A ototoxicidade é em geral considerada irreversível, mas alguns estudos sugerem que o efeito sobre o ouvido interno possa ser reversível.

Bernard et al. ${ }^{(15)}$ realizaram um estudo com 70 pacientes portadores de AR e 4 portadores de LES, em uso de FC, sendo que 5 pacientes apresentavam perda neurossensorial moderada antes do início do tratamento, porém nenhum com sintomas vestibulares. Anormalidades no BERA (Brainstem evoked response audiometry) tornaram-se evidentes em 13 pacientes por volta do oitavo mês de tratamento, todos com AR. Nesse estágio, apenas dois pacientes relataram episódios de zumbido e desequilíbrio ${ }^{(15)}$. A terapia com FC foi permanentemente suspensa em 12 dos 13 pacientes. Destes 12 pacientes, o BERA permaneceu anormal em 9 e houve piora em três pacientes nos quatro meses subseqüentes, ocorrendo retorno aos valores iniciais num período de 12 a 16 meses, posteriormente estabilizado. Esses pacientes não apresentaram em momento algum alterações na audiometria ou no exame oftalmológico. Em um caso foi indicada continuação da terapia com FC, por apresentar fortes dores e o BERA foi progressivamente deteriorando, ocorrendo perda auditiva permanente ${ }^{(15)}$.

Johansen e Gran ${ }^{(16)}$ relataram o primeiro caso de ototoxicidade com uso de HCQ, em um paciente com LES e outro com lúpus discóide em uso da droga há vários anos. Ambos desenvolveram perda neurossensorial severa bilateral irreversível. 
Seçkin ${ }^{(17)}$ relata um caso de ototoxicidade com HCQ em paciente com AR na dose de $400 \mathrm{mg} /$ dia por cinco meses, desenvolvendo disacusia neurossensorial leve bilateral e zumbidos. Após interrupção do tratamento apresentou melhora dos zumbidos em duas semanas e o audiograma retornou ao normal em dois meses.

Coutinho e Duarte ${ }^{(4)}$ relatam um caso de paciente de sete anos, com hemossiderose pulmonar que evoluiu com perda auditiva neurossensorial de moderada a severa sem acometimento vestibular após dois anos de uso de HCQ na dose de $200 \mathrm{mg} /$ dia. Seu tratamento não foi interrompido por ser uma droga vital ao controle da doença. Em um trabalho recente, realizado na Universidade de São Paulo ${ }^{(18)}$, não foram observados sinais de ototoxicidade infantil avaliada por audiometria tonal limiar em nove crianças maiores de quatro anos de idade expostas in utero ao FC, em comparação com outras 10 crianças cujas mães não utilizaram FC seis meses antes da concepção.

O objetivo desta revisão foi alertar para o potencial ototóxico dos antimaláricos, diretamente relacionado com o

\section{REFERÊNCIAS}

1. Dauman R, Dulon D: Ototoxicidad medicamentosa. Encyclopedie Médico-Chirurgicale, 20:184-B-0, 1995.

2. Trehan M: The use of antimalarials in dermatology. J Dermatol Treat 11: 185-94, 2000.

3. Usamah H, Nuwayhid N, Hasbini AS: Chloroquine ototoxicity: An idiosyncratic phenomenon. Otolaryngol Head Neck Surg 114: 491-3, 1996.

4. Coutinho MB, Duarte I: Hydroxychloroquine ototoxicity in a child with idiopathic pulmonary haemosiderosis. Int J Pediatr Otorhinolaryngol 11 62: 53-7, 2002.

5. Clark P, Casas E, Tugwell P, et al: Hydroxychloroquine compared to placebo in rheumatoid arthritis. A randomized controlled trial. Ann Intern Med 119: 1067-71,1996.

6. Dencker L, Lindquist NG: Distribution of labeled chloroquine in the inner Ear. Arch Otolaryngology 101: 185-8,1975.

7. Murkherjee DK, Enugu FRCS: Chloroquine ototoxicity - a reversible phenomenon? J Laryngol Otol 93: 809-15, 1979.

8. Hart CW, Naunton RF: The ototoxicity of chloroquine phosphate. Arch Otolaryngol 80: 407-12, 1964.

9. Matz GJ, Naunton RF: Ototoxicity of chloroquine. Arch Otolaryngology 88: 370-2, 1968.

10. Buchanan NM, Toubi E, Khamashta MA, Lima F, Kerslake S, Hughes GR: Hydroxychloroquine and lupus pregnancy: review of a series of 36 cases. Ann Rheum Dis 55: 486-8, 1996. tipo e dose da substância utilizada (FC ou HCQ). com a sensibilidade do paciente e com o tempo de uso. Destacamse algumas conclusões:

- O FC provoca efeitos ototóxicos, que podem ser reversíveis se a medicação for suspensa em tempo hábil.

- Com o uso de HCQ a ototoxicidade é extremamente rara.

- O FC pode ser ototóxico para fetos, se usado por gestantes no terceiro trimestre da gestação; já a HCQ não causa danos e é utilizada.

- Perda neurossensorial profunda irreversível pode seguir o tratamento continuado com FC.

- A ototoxicidade está ligada à destruição de células sensoriais, diminuição na população neuronal, alterações nas células de sustentação e atrofia da estria vascular; tais alterações podem decorrer da isquemia.

Portanto, sugerimos que se considere a avaliação audiológica periódica de rotina dos pacientes em uso regular de FC, para que possam ser detectadas precocemente eventuais alterações ototóxicas, evitando-se, assim, possíveis danos irreversíveis.

11. Motta M, Tincani A, Faden D, Zinzini E, Chirico G: Antimalarial agents in pregnancy. Lancet 359: 524-5, 2002.

12. Costedoat-Chalumeau N, Amoura Z, Duhaut P, et al: Safety of hydroxychloroquine in pregnant patients with connective tissue diseases: a study of one hundred thirty-three cases compared with a control group. Arthritis Rheum 48: 3207-11, 2003

13. Chakravarty EF, Sanchez-Yamamoto D, Bush TM. The Use of Disease-modifying Antirheumatic Drugs in Women with Rheumatoid Arthritis of Childbearing Age: A Survey of Practice Patterns and Pregnancy Outcomes. J Rheumatol 30: 241-6, 2003.

14. Levy RA, Vilela VS, Cataldo MJ, et al: Hydroxychloroquine (HCQ) in lupus pregnancy: double blind and placebo controlled study. Lupus 10: 401-4, 2001.

15. Bernard P: Alterations of auditory evoked potentials during the course of chloroquine treatment. Acta Otolaryngol 99: 387-92, 1985.

16. Johansen PB, Gran JT: Ototoxicity due to hydroxychloroquine: report of two cases. Clin Exp Rheumatol 164: 472-4, 1998

17. Seçkin Ü, Özoran K, Ikinciogullari A, Borman P, Bostan EE: Hydroxychloroquine ototoxicity in a patient with rheumatoid arthritis. Rheumatol Int 19: 203-4, 2000.

18. Turrini-Filho JR, Kuruma KAM, Bertola C, Pedalini MEB, Borba EF, Bonfá ESD: Lack Of Child Ototoxicity By Limiar Tonal Audiometry (lta) After chloroquine gestacional use in systemic lupus erythematosus. Arthritis Rheum S180, 2003. 